\title{
Circulating SIRT1 inversely correlates with epicardial fat thickness in patients with obesity
}

\author{
S. Mariani*, D. Costantini, C. Lubrano, S. Basciani, C. Caldaroni, G. Barbaro, \\ E. Poggiogalle, L.M. Donini, A. Lenzi, L. Gnessi \\ Department of Experimental Medicine, Section of Medical Physiopathology, Food Science and Endocrinology, "Sapienza" University of Rome, 00161 \\ Rome, Italy \\ Received 19 February 2016; received in revised form 25 May 2016; accepted 8 June 2016 \\ Available online 16 June 2016
}

\section{KEYWORDS \\ SIRT1; \\ Epicardial fat; Obesity}

\begin{abstract}
Background and aim: Obesity is increasing worldwide and is related to undesirable cardiovascular outcomes. Epicardial fat (EF), the heart visceral fat depot, increases with obesity and correlates with cardiovascular risk. SIRT1, an enzyme regulating metabolic circuits linked with obesity, has a cardioprotective effect and is a predictor of cardiovascular events. We aimed to assess the relationship of EF thickness (EFT) with circulating SIRT1 in patients with obesity. Methods and results: Sixty-two patients affected by obesity and 23 lean controls were studied. Plasma SIRT1 concentration was determined by enzyme-linked immunosorbent assay (ELISA). EFT was measured by echocardiography. Body mass index (BMI), waist circumference, heart rate (HR), blood pressure, and laboratory findings (fasting glucose, insulin, HbA1c, cholesterol, and triglycerides) were assessed.

SIRT1 was significantly lower $(P=0.002)$ and EFT was higher $(P<0.0001)$ in patients with obesity compared with lean controls. SIRT1 showed a negative correlation with EFT and HR in the obesity group ( $\rho=-0.350, P=0.005 ; \rho=-0.303, P=0.008$, respectively). After adjustment for obesity-correlated variables, multiple linear regression analysis showed that EFT remained the best correlate of SIRT1 $(\beta=-0.352, P=0.016)$.

Conclusions: Circulating SIRT1 correlates with the visceral fat content of the heart. Serum SIRT1 levels might provide additional information for risk assessment of coronary artery disease in patients with obesity.

( 2016 The Italian Society of Diabetology, the Italian Society for the Study of Atherosclerosis, the Italian Society of Human Nutrition, and the Department of Clinical Medicine and Surgery, Federico II University. Published by Elsevier B.V. All rights reserved.
\end{abstract}

\section{Introduction}

Sirtuins (SIRTs) regulate many metabolic adaptations in obesity [1]. SIRT1, the most intensely studied sirtuin family member, regulates the expression of adipokines, represses the activity of factors required for maturation of fat cells, alters mitochondrial capacity, regulates insulin secretion and sensitivity, and modulates plasma glucose levels [1]. In

\footnotetext{
* Corresponding author. Tel.: +39 6 49970721; fax: +39 64461450 .

E-mail address: stefaniamariani@yahoo.com (S. Mariani).
}

addition, SIRT1 prevents diet-induced obesity and associated non-alcoholic fatty liver disease [2], a condition of ectopic fat accumulation accompanied by a decrease in SIRT1 expression at both circulating [3] and visceral adipose tissue (VAT) levels [4]. In line with these observations, the weight loss induces a SIRT1 concentration increase in plasma [5], adipose tissue, and liver [6] in patients with obesity. Overall, SIRT1 is found to be protective against endothelial dysfunction, atherothrombosis, myocardial infarction [2], and cardiovascular diseases in general. Indeed, by controlling the cardiac metabolic gene expression through modulation of the peroxisome 
proliferator activated receptor $\alpha(\operatorname{PPAR} \alpha)$ pathway, SIRT1 protects the heart from hypertrophy, metabolic dysregulation, and inflammation [7,2].

Epicardial fat (EF), the adipose tissue of the heart, is a VAT. It covers $80 \%$ of the heart's surface and, because of its anatomical contiguity with the heart, locally modulates the functions of myocardium and coronary arteries through a paracrine cross talk [8-10]. EF has potential cardioprotective effects that can be exerted mechanically, thermogenically, and throughout the local secretion of adipokines [8]. However, excess EF carries a great prediction of mortality [11]. Specifically, EF thickness (EFT) is associated with premature coronary artery disease [12] and obstructive sleep apnea [13]. EFT increases in prediabetic patients, regardless of fasting plasma glucose and HbA1c [14], and in patients with type 2 diabetes mellitus and obesity [15], representing a diagnostic criterion for patients with metabolic syndrome [16]. It is also inversely correlated with endothelial function [17]. In this study, we focused on the association of circulating SIRT1 levels with the visceral fat of the heart, represented by EF, in patients affected by obesity.

\section{Methods}

\section{Study population}

We studied 85 subjects, 62 affected by obesity (19 men and 43 women) and 23 healthy lean controls ( 7 men and 19 women). Study participants were recruited from among subjects referred to the High Specialization Center for the Care of Obesity at the Department of Experimental Medicine, "Sapienza" University of Rome. Inclusion criteria comprised age (18-65 years), race (Caucasian ethnicity), BMI $\geq 30 \mathrm{~kg} / \mathrm{m}^{2}$ for obese patients, and BMI $\geq 18.5$ and $\leq 24.9 \mathrm{~kg} / \mathrm{m}^{2}$ for controls. As exclusion criteria, we considered corticosteroids for systemic use, any medication, or any clinical condition potentially affecting body weight, autoimmune diseases, renal failure, heart failure, type 1 diabetes, and malignant disease during the last 5 years.

The average age was $39.58 \pm 12.27$ years (range $18-65$ years) for subjects with obesity and $41.69 \pm 10.84$ years (range 22-59 years) for controls. Subjects affected by obesity and previously diagnosed with diabetes mellitus, dyslipidemia, or hypertension were receiving glucose-, lipid-, and BP-lowering agents for each of these conditions. All subjects were enrolled after written informed consent. The study was approved by the local ethical committee and was concordant with the Helsinki Declaration.

\section{Measurements}

The patients underwent complete medical examination and anthropometric measurements (body weight, height, waist circumference (WC)). Body weight was measured by Tanita BWB-800A digital medical scale (Tanita Corporation, Arlington Heights, IL, USA). Body mass index (BMI) was calculated as body weight $(\mathrm{kg})$ divided by height squared $\left(\mathrm{m}^{2}\right)$. After overnight fasting, serum SIRT1, glycemia, insulin, total cholesterol, low-density lipoproteincholesterol (LDL-C), high-density lipoprotein-cholesterol (HDL-C), and triglycerides were determined. Heart rate (HR) and systolic and diastolic blood pressure ( $\mathrm{mmHg}$ ) measured by a mercury sphygmomanometer (Riva-Rocci System, ERKA, Chemnitz, Germany) were recorded. EFT $(\mathrm{mm})$ was measured by ultrasound.

\section{SIRT1 assays}

The plasma SIRT1 concentration was determined by a monoclonal antibody-based enzyme-linked immunosorbent assay (ELISA) method, using a commercially available human SIRT1 ELISA kit (MyBioSource, Cod. GDMBS 705558) as previously described [3]. Microtiter plates were coated with equal amount of primary mouse anti-human SIRT1 monoclonal IgG. Hundred-microliter standard and serum samples were pipetted in each well and the protocol was followed by using secondary avidin conjugated with horseradish peroxidase. The formation of horseradish peroxidase was measured at $405 \mathrm{~nm}$ using an ELISA reader (Quanta Biotech, UK). Seven different concentrations of purified SIRT1 $(0.15,0.312,0.625,1.25,2.5,5.0$, and $10 \mathrm{ng} /$ $\mathrm{mL}$ ) were used to plot a standard curve. The inter- and intra-assay coefficients of variation were $4 \%$ and $6 \%$, respectively, with a detection limit of $0.1 \mathrm{ng} / \mathrm{mL}$.

\section{Epicardial fat thickness measurements}

EFT was measured through a validated echocardiographic procedure [18]. Participants underwent highresolution M-B-mode transthoracic echocardiography using a 2.5-MHz probe and spectral Doppler exam of the common carotid artery using a $7.5-\mathrm{MHz}$ probe (Esaote MyLab40, Esaote Europe B.V., the Netherlands). The EFT was identified as the echo-free space between the outer wall of the myocardium and the visceral layer of the pericardium, and its thickness was measured perpendicularly on the free wall of the right ventricle (RV) at the end systole in three cardiac cycles. The average value of three cardiac cycles from each echocardiographic view was considered. All echocardiograms were recorded by the same experienced operator, who was blinded to the other study data.

\section{Statistical analysis}

Results were expressed as mean \pm SD. The degree of association between variables was calculated using Spearman's nonparametric correlation. A $p$-value of $<0.05$ was considered statistically significant. Sex- and age-adjusted multivariate regression analyses were realized in subjects with obesity to verify the associations among SIRT1 and relevant variables (EFT, HR, BMI, WC, and HbA1c). Data were analyzed with the use of STATISTICA software, version 6.1 (StatSoft, Inc., Tulsa, OK, USA). 


\section{Results}

The demographic, anthropometric, and clinical characteristics of the patients are shown in Table 1 . The patients with obesity (BMI, mean \pm SD $41.82 \pm 7.67 \mathrm{~kg} / \mathrm{m}^{2}$ ) showed echocardiographic evidence of increase in EFT $(P<0.0001)$ and significantly lower SIRT1 circulating levels $(P=0.002)$ compared with normal-weight subjects. The WC was constantly $\geq 80 \mathrm{~cm}$ in females and $\geq 94 \mathrm{~cm}$ in males with obesity. The group of healthy lean controls (BMI, mean \pm SD $23.26 \pm 1.60 \mathrm{~kg} / \mathrm{m}^{2}$ ) had no ultrasound evidence of increase in EFT. The total cholesterol $(P=0.045)$, LDL-C $(P=0.040)$, systolic BP $(P=0.041)$, diastolic BP $(P=0.011)$, and insulin $(P=0.020)$ levels were significantly lower in the population of lean controls (Table 1 ).

The correlation between SIRT1 and the other variables was analyzed using the Spearman correlation coefficient test. In the population with obesity, SIRT1 was negatively correlated with EFT $(\rho=-0.350 ; P=0.005)$ (see Fig. 1 ) and HR $(\rho=-0.303 ; P=0.008)$. On the contrary, SIRT1 did not correlate with BMI $(P=0.326)$, WC $(P=0.263)$, FPG $(P=0.415)$, and the other parameters tested. A tendency to a negative correlation with HbA1c was also seen ( $\rho=-0.216 ; P=0.062$ ). In the healthy lean control group, SIRT1 did not correlate with any of the parameters measured (data not shown).

Sex- and age-adjusted multivariate regression analyses for the associations between SIRT1 and other relevant variables (EFT, HR, BMI, WC, and HbA1c) in patients with obesity are shown in Table 2 . The analysis revealed a significant negative correlation between the levels of SIRT and the EFT, which remained the best independent correlate of SIRT1 $\left(R=0.429, R^{2}=0.184, \beta=-0.352\right.$, $P=0.016)$.

Table 1 Demographic, anthropometric, and clinical characteristics of the patients.

\begin{tabular}{lccc}
\hline Variables & $\begin{array}{c}\text { Obese subjects } \\
(n=62)\end{array}$ & $\begin{array}{c}\text { Normal weight } \\
(n=23)\end{array}$ & $P$ \\
\hline Age (years) & $39.58 \pm 12.27$ & $41.69 \pm 10.84$ & 0.439 \\
SIRT1 $(\mathrm{ng} / \mathrm{ml})$ & $1.36 \pm 1.31$ & $2.27 \pm 1.13$ & 0.002 \\
Weight $(\mathrm{kg})$ & $116.17 \pm 24.36$ & $65.09 \pm 7.07$ & $<0.0001$ \\
BMI $\left(\mathrm{kg} / \mathrm{m}^{2}\right)$ & $41.82 \pm 7.67$ & $23.26 \pm 1.60$ & $<0.0001$ \\
WC $(\mathrm{cm})$ & $126.21 \pm 14.71$ & $78.80 \pm 11.91$ & $<0.0001$ \\
Epicardial fat $(\mathrm{mm})$ & $8.49 \pm 0.87$ & $6.97 \pm 0.57$ & $<0.0001$ \\
Heart rate $(\mathrm{bpm})$ & $73.58 \pm 8.53$ & $74.30 \pm 6.44$ & 0.695 \\
FPG $(\mathrm{mg} / 100 \mathrm{ml})$ & $100.57 \pm 21.42$ & $98.65 \pm 16.15$ & 0.677 \\
Insulin $(\mathrm{mcUl} / \mathrm{ml})$ & $16.48 \pm 14.04$ & $9.88 \pm 3.95$ & 0.020 \\
HbA1c $(\%)$ & $5.05 \pm 0.62$ & $4.77 \pm 0.92$ & 0.089 \\
Systolic BP $(\mathrm{mmHg})$ & $127.36 \pm 14.51$ & $120.57 \pm 14.09$ & 0.041 \\
Diastolic BP $(\mathrm{mmHg})$ & $79.34 \pm 10.46$ & $73.57 \pm 7.43$ & 0.011 \\
Total-C $(\mathrm{mg} / 100 \mathrm{ml})$ & $197.69 \pm 33.45$ & $183.42 \pm 21.92$ & 0.045 \\
LDL-C $(\mathrm{mg} / 100 \mathrm{ml})$ & $120.68 \pm 28.17$ & $107.66 \pm 25.71$ & 0.040 \\
HDL-C $(\mathrm{mg} / 100 \mathrm{ml})$ & $48.66 \pm 12.90$ & $49.30 \pm 15.22$ & 0.835 \\
Triglycerides & $132.81 \pm 82.86$ & $108.36 \pm 50.61$ & 0.160 \\
\multicolumn{1}{c}{ (mg/100 ml) } & & & \\
\hline Abbrevitions: SlRTl, & & & \\
\hline
\end{tabular}

Abbreviations: SIRT1, sirtuin1; BMI, body mass index; WC, waist circumference; FPG, fasting plasma glucose; HbA1c, glycated hemoglobin; BP, blood pressure; HDL-C, high-density lipoproteincholesterol; LDL-C, Low-density lipoprotein-cholesterol. Values are expressed as means $\pm \mathrm{SD}$.

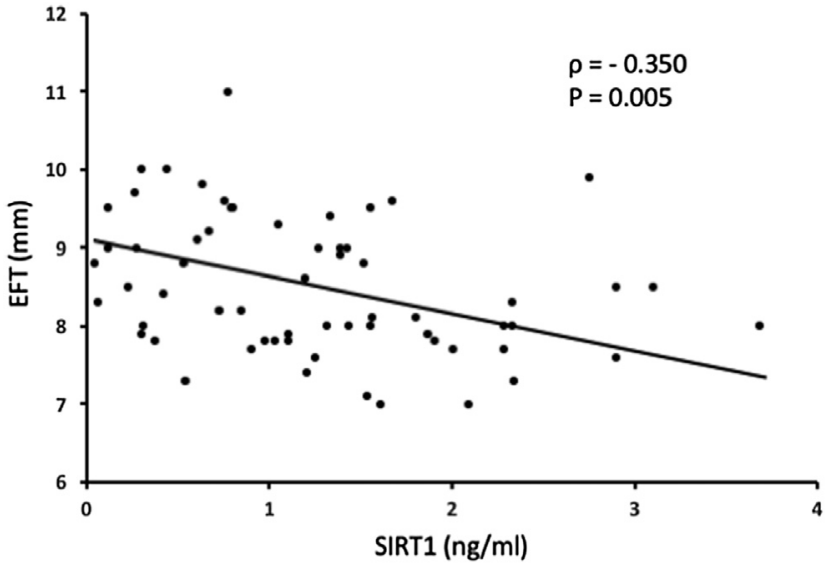

Figure 1 Correlation between circulating SIRT1 and epicardial fat thickness in people with obesity. Correlation coefficient $(\rho)$ and level of significance $(P)$ are provided.

According to previous studies [19], a correlation between EFT and both BMI $(\rho=0.617, P<0.0001)$ and WC ( $\rho=0.640, P<0.0001)$ was found.

\section{Discussion}

VAT, including the EF surrounding the heart, shows many histological and functional peculiarities. VAT contains a large number of inflammatory cells and expresses several hormone receptors, and its adipocytes are metabolically active and sensitive to lipolysis. The increased amount of VAT carries, in general, a great prediction of mortality [11] and $\mathrm{EF}$, which substantially mirrors visceral adiposity rather than general obesity, has been correlated with cardiovascular risk factors $[8,13,14]$. EFT increases in obese patients. It is a useful parameter in the assessment of patients with obesity and other cardio-metabolic diseases [17]. Due to its proximity to the heart, by secreting proinflammatory cytokines EF exerts direct local metabolic effects. This condition promotes local cardiovascular diseases and contributes, together with other ectopic fat depots, to metabolic derangements and diabetes mellitus [20].

SIRT1, a (NAD+)-dependent enzyme, is significantly involved in the metabolic adaptations observed in obesity

Table 2 Age- and sex-adjusted multivariate regression analysis of SIRT1.

\begin{tabular}{lll}
\hline Independent variables & $\beta$ & $\mathrm{p}$ \\
\hline EFT $(\mathrm{mm})$ & -0.352 & 0.016 \\
HR $(\mathrm{bpm})$ & -0.133 & 0.316 \\
BMI $\left(\mathrm{kg} / \mathrm{m}^{2}\right)$ & 0.134 & 0.557 \\
WC $(\mathrm{cm})$ & -0.096 & 0.689 \\
HbA1c $(\%)$ & -0.157 & 0.220
\end{tabular}

Abbreviations: SIRT1, sirtuin1; EFT, epicardial fat thickness; HR, heart rate; BMI, body mass index; WC, waist circumference; HbA1c, glycated hemoglobin. EFT, HR, BMI, WC, and HbA1c were entered in the multiple regression analysis to predict SIRT1, the dependent variable. EFT was the best independent correlate of SIRT1 in patients with obesity. 
[1]. Most studies report low SIRT1 in subjects affected by obesity and higher SIRT1 expression after weight loss, both in tissues [6] and in bloodstream [5], suggesting that SIRT1 is downregulated in obesity. The awareness of the role of SIRT1 against metabolic derangements such as diabetes, dyslipidemia, and liver steatosis has been increasing [21, [22]. This role is further highlighted by the observation of a low SIRT1 pathway expression in subcutaneous adipose tissue from a population of BMI-discordant monozygotic twins [23] affected by obesity, inflammation, insulin resistance, and impaired mitochondrial protein homeostasis. This datum, besides suggesting a strong relationship of reduced SIRT1 expression with indices of metabolic dysfunction, indicates a close relationship between SIRT1 and acquired obesity independent of the genetic background [23].

To date, substantial evidence suggests a role of SIRT1 in cardioprotection [2]. The relevance of SIRT1 as a cardiac gene regulator is well established, and low SIRT1 expression in the context of cardiovascular disease has been reported [7]. Furthermore, SIRT1 shows a significant contribution in oxidant and antioxidant balance in heart failure [24] and, via activating eNOS, its overexpression protects against myocardial ischemia-reperfusion injury in diabetic rats, representing a promising therapeutic target for cardiac complications in diabetics [25]. Finally, the SIRT1 gene KO leads to cardiac malformations and increased perinatal mortality in mice [26].

In this study, we evaluated the relationship between SIRT1 and the visceral fat depot of the heart. We found that EFT is a strong negative correlate of the circulating SIRT1 in individuals affected by obesity. This behavior closely resembles that of other bioactive compounds produced by EF with reported cardioprotective effects, both in vivo and in vitro. For example, the increase in EFT is accompanied by a reduced expression of adiponectin [27], decreased circulating adiponectin levels in genetic and diet-induced murine models of obesity, and downregulated EF adrenomedullin gene and protein expression in subjects with coronary artery disease [28]. Thus, SIRT1, analogously to bioactive adipokines originating from $\mathrm{EF}$, shows a correlation with cardio-metabolic status and amount of $\mathrm{EF}$. Accordingly, EF revealed a unique transcriptome that shows profound modifications in patients with coronary artery disease [29], and among the differentially expressed genes in human EF to identify molecules associated with cardiovascular diseases, SIRT1 was represented [30]. Consistent with previous studies [3,5], we did not find a relationship between circulating SIRT1 and BMI or WC, although an association among EFT, BMI, and WC was seen. This observation confirms that the increase in BMI and WC may not reflect a reduction in SIRT1, which is a distinctive trait of individuals mostly with visceral obesity, nor may it be representative of the excess visceral fat depot responsible for the severity of cardio-metabolic complications of obesity. Furthermore, since regional fat depots may be of greater importance than overall adiposity, traditional anthropometric measures should be viewed with caution. Our finding highlights the poor relationship between SIRT1 and the classic measures of adiposity. Indeed, analogously to the recent observation of a negative association between SIRT1 and liver steatosis [3], a condition of ectopic fat accumulation, we found a significant correlation between SIRT1 and EF, the ectopic visceral fat depot of the heart whose major drive is not obesity but excessive visceral fat accumulation [8]. Thus, SIRT1 is preferentially associated with visceral fat rather than with obesity per se. From this point of view, SIRT1 could be elevated to the rank of marker associated with the predisposition to accumulate fat viscerally. It is noteworthy that in normal-weight patients, who had higher SIRT1 concentration and lower EFT than patients with obesity did, we observed that the correlation between SIRT1 and EFT was lacking. Interestingly, myocardial dysfunction seems to be related to scarce metabolic control rather than to fat mass or BMI [31]. Previous data have suggested that patients with obesity yet metabolically healthy carry a reduced risk of cardiovascular disease or mortality than patients with normal weight yet metabolically unhealthy [32]. However, the definition of metabolically healthy obesity is established using BMI, an improper index of adiposity. Therefore, SIRT1 measurement could help in stratifying cardiovascular disease risk and identifying metabolically healthy or unhealthy obese phenotypes. The exact mechanisms involved in the inverse relationship between circulating SIRT1 and EFT reported here are unknown. Mounting evidence shows that SIRT transcription and/or protein levels are persistently reduced in specific tissue during chronic inflammation. Examples include fat deposits in obesity with inflammation, brain in Alzheimer's disease, and arterial inflammation in atherosclerosis [33]. Thus, a working hypothesis, which deserves further investigation, is that excess $E F$ in patients with visceral obesity displays a pro-inflammatory phenotype; an oxidative stress environment is created, mainly due to a state of chronic inflammation prone to secreting tumor necrosis factor alpha (TNF- $\alpha$ ), interleukin (IL)-6, and inducible nitric oxide synthase (iNOS), with heightened inflammation associated with low SIRT1 and expansion of adipose tissue.

Although EF may be a major source of circulating SIRT1, because of the widespread distribution of the potential tissue sources of SIRT1 [21], additional sources of circulating SIRT1, besides EF, cannot be ruled out.

Unexpectedly, SIRT1 correlated with the HR in patients with obesity. The exact mechanism for such an association is unclear, and this aspect of the study deserves further investigation. However, the diversity of SIRT1 targets may explain the complexity of its function in different tissues, including the cardiac electric tissue. Consistently, increased SIRT1 expression is found in the right auricle tissues of patients with atrial fibrillation (AF) [34]. Moreover, $\mathrm{EF}$ is significantly thicker in individuals with chronic $\mathrm{AF}$ than in those with paroxysmal AF, suggesting that longterm exposure to $\mathrm{EF}$ excess might relate to chronic $\mathrm{AF}$ [35].

Finally, recent data showed that PDE5 inhibitor treatment in humans reduces EF and upregulates SIRT1 in both serum and subcutaneous fat, opening novel therapeutic 
strategies for EF remodeling and regulation of SIRT1 to promote healthier fat deposits [36].

\section{Conclusion}

In conclusion, the findings of this study suggest that in people with obesity, SIRT1, a metabolically crucial enzyme, is inversely and independently associated with EFT, but no relationship between circulating SIRT1 and BMI or WC was seen. A large body of evidences suggests a role of SIRT1 as a predictor of cardiovascular events, and our present data seem to go in the same direction. Overall, the downregulation of SIRT1 expression observed at the circulating level and in visceral and subcutaneous adipose tissues of people with obesity [37], paralleled by the increase in epicardial VAT, may contribute to the cardio-metabolic abnormalities associated with obesity. This field will be further investigated in the light of the disposability of SIRT1 mimetics/activators able to increase SIRT1 activities.

\section{Disclosures}

The authors declared no conflict of interest.

\section{Acknowledgments}

This work was supported by the Sapienza University of Rome, Italy. The funding source had no involvement in study design; in the collection, analysis, and interpretation of data; in the writing of the report; and in the decision to submit the article for publication.

\section{References}

[1] Metoyer CF, Pruitt K. The role of sirtuin proteins in obesity. Pathophysiology 2008;15(2):103-8. http://dx.doi.org/10.1016/ j.pathophys.2008.04.002.

[2] Winnik S, Auwerx J, Sinclair DA, Matter CM. Protective effects of sirtuins in cardiovascular diseases: from bench to bedside. Eur Heart J 2015;36(48):3404-12. http://dx.doi.org/10.1093/ eurheartj/ehv290.

[3] Mariani S, Fiore D, Basciani S, Persichetti A, Contini S, Lubrano C, et al. Plasma levels of SIRT1 associate with non-alcoholic fatty liver disease in obese patients. Endocrine 2015;49(3):711-6. http: //dx.doi.org/10.1007/s12020-014-0465-x.

[4] Costa Cdos S, Hammes TO, Rohden F, Margis R, Bortolotto JW, Padoin AV, et al. SIRT1 transcription is decreased in visceral adipose tissue of morbidly obese patients with severe hepatic steatosis. Obes Surg 2010;20:633-9. http://dx.doi.org/10.1007/s11695009-0052-Z.

[5] Mariani S, Fiore D, Persichetti A, Basciani S, Lubrano C, Poggiogalle E, et al. Circulating SIRT1 increases after intragastric balloon fat loss in obese patients. Obes Surg 2016 Jun;26(6): 1215-20. http://dx.doi.org/10.1007/s11695-015-1859-4.

[6] Moschen AR, Wieser V, Gerner RR, Bichler A, Enrich B, Moser P, et al. Adipose tissue and liver expression of SIRT1, 3, and 6 increase after extensive weight loss in morbid obesity. J Hepatol 2013; 59(6):1315-22. http://dx.doi.org/10.1016/j.jhep.2013.07.027.

[7] Planavila A, Iglesias R, Giralt M, Villarroya F. Sirt1 acts in association with PPAR\{alpha\} to protect the heart from hypertrophy, metabolic dysregulation, and inflammation. Cardiovasc Res 2011; 90:276-84. http://dx.doi.org/10.1093/cvr/cvq376.

[8] Iacobellis G. Local and systemic effects of the multifaceted epicardial adipose tissue depot. Nat Rev Endocrinol 2015;11(6): 363-71. http://dx.doi.org/10.1038/nrendo.2015.58.
[9] Mahabadi AA, Massaro JM, Rosito GA, Levy D, Murabito JM, Wolf PA, et al. Association of pericardial fat, intrathoracic fat, and visceral abdominal fat with cardiovascular disease burden: the Framingham Heart Study. Eur Heart J 2009;30:850-6. http: //dx.doi.org/10.1093/eurheartj/ehn573.

[10] Katsiki N, Mikhailidis DP, Wierzbicki AS. Epicardial fat and vascular risk: a narrative review. Curr Opin Cardiol 2013;28: 458-63. http://dx.doi.org/10.1097/HCO.0b013e3283605fba.

[11] Ibrahim MM. Subcutaneous and visceral adipose tissue: structural and functional differences. Obes Rev 2010;11(1):11-8. http: //dx.doi.org/10.1111/j.1467-789X.2009.00623.x.

[12] Faghihi S, Vasheghani-Farahani A, Parsaee M, Saedi S, Ghadrdoost B. Association between epicardial fat thickness and premature coronary artery disease: a case control study. Res Cardiovasc Med 2015;4(2):e25679. http://dx.doi.org/10.5812/cardiovascmed.4(2)2015.25679.

[13] Mariani S, Fiore D, Barbaro G, Basciani S, Saponara M, D'Arcangelo E, et al. Association of epicardial fat thickness with the severity of obstructive sleep apnea in obese patients. Int J Cardiol 2013;167(5):2244-9. http://dx.doi.org/10.1016/ j.ijcard.2012.06.011.

[14] Arpaci D, Ugurlu BP, Aslan AN, Ersoy R, Akcay M, Cakir B. Epicardial fat thickness in patients with prediabetes and correlation with other cardiovascular risk markers. Intern Med 2015;54(9): 1009-14. http://dx.doi.org/10.2169/internalmedicine.54.3714.

[15] Song DK, Hong YS, Lee H, Oh JY, Sung YA, Kim Y. Increased epicardial adipose tissue thickness in type 2 diabetes mellitus and obesity. Diabetes Metab J 2015;39(5):405-13. http: //dx.doi.org/10.4093/dmj.2015.39.5.405.

[16] Kaya B, Kaya BC, Karakas EY, Baysal SS, Cadirci D, Erkus E, et al. Usefulness of the epicardial fat tissue thickness as a diagnostic criterion for geriatric patients with metabolic syndrome. J Geriatr Cardiol 2015;12(4):373-7. http://dx.doi.org/10.11909/j.issn.16715411.2015.04.008.

[17] Aslan AN, Keleş T, Ayhan H, Kasapkara HA, Akçay M, Durmaz T, et al. The relationship between epicardial fat thickness and endothelial dysfunction in type I diabetes mellitus. Echocardiography 2015;32(12):1745-53. http://dx.doi.org/10.1111/echo.12960.

[18] Malavazos AE, Di Leo G, Secchi F, Lupo EN, Dogliotti G, Coman C, et al. Relation of echocardiographic epicardial fat thickness and myocardial fat. Am J Cardiol 2010;105:1831-5. http: //dx.doi.org/10.1016/j.amjcard.2010.01.368.

[19] Rabkin SW. The relationship between epicardial fat and indices of obesity and the metabolic syndrome: a systematic review and meta-analysis. Metab Syndr Relat Disord 2014;12(1):31-42. http: //dx.doi.org/10.1089/met.2013.0107.

[20] Noyes AM, Dua K, Devadoss R, Chhabra L. Cardiac adipose tissue and its relationship to diabetes mellitus and cardiovascular disease. World J Diabetes 2014;5(6):868-76. http: //dx.doi.org/10.4239/wjd.v5.i6.868.

[21] Chang HC, Guarente L. SIRT1 and other sirtuins in metabolism. Trends Endocrinol Metab 2014;25(3):138-45. http: //dx.doi.org/10.1016/j.tem.2013.12.001.

[22] Boutant M, Cantó C. SIRT1 metabolic actions: integrating recent advances from mouse models. Mol Metab 2013;3(1):5-18. http: //dx.doi.org/10.1016/j.molmet.2013.10.006. eCollection 2014.

[23] Jukarainen S, Heinonen S, Rämö JT, Rinnankoski-Tuikka R, Rappou E, Tummers M, et al. Obesity is associated with low $\mathrm{NAD}(+) /$ SIRT pathway expression in adipose tissue of BMIdiscordant monozygotic twins. J Clin Endocrinol Metab 2016; 101(1):275-83. http://dx.doi.org/10.1210/jc.2015-3095.

[24] Akkafa F, Halil Altiparmak I, Erkus ME, Aksoy N, Kaya C, Ozer A, et al. Reduced SIRT1 expression correlates with enhanced oxidative stress in compensated and decompensated heart failure. Redox Biol 2015;6:169-73. http://dx.doi.org/10.1016/ j.redox.2015.07.011.

[25] Ding M, Lei J, Han H, Li W, Qu Y, Fu E, et al. SIRT1 protects against myocardial ischemia-reperfusion injury via activating eNOS in diabetic rats. Cardiovasc Diabetol 2015;14(1):143. http: //dx.doi.org/10.1186/s12933-015-0299-8.

[26] Cheng HL, Mostoslavsky R, Saito S, Manis JP, Gu Y, Patel P, et al. Developmental defects and p53 hyperacetylation in Sir2 homolog (SIRT1)-deficient mice. Proc Natl Acad Sci USA 2003;100:10794-9.

[27] Dozio E, Vianello E, Briganti S, Lamont J, Tacchini L, Schmitz G, et al. Expression of the receptor for advanced glycation end 
products in epicardial fat: link with tissue thickness and local insulin resistance in coronary artery disease. J Diabetes Res 2016; 2016:2327341. http://dx.doi.org/10.1155/2016/2327341.

[28] Iacobellis G, di Gioia CR, Di Vito M, Petramala L, Cotesta D, De Santis V, et al. Epicardial adipose tissue and intracoronary adrenomedullin levels in coronary artery disease. Horm Metab Res 2009;41(12):855-60. http://dx.doi.org/10.1055/s-0029-1231081.

[29] McAninch EA, Fonseca TL, Poggioli R, Panos AL, Salerno TA, Deng Y et al. Epicardial adipose tissue has a unique transcriptome modified in severe coronary artery disease. Obesity 2015;23(6): 1267-78. http://dx.doi.org/10.1002/oby.21059.

[30] Maghbooli Z, Hossein-Nezhad A. Transcriptome and molecular endocrinology aspects of epicardial adipose tissue in cardiovascular diseases: a systematic review and meta-analysis of observational studies. Biomed Res Int 2015;2015:926567. http: //dx.doi.org/10.1155/2015/926567.

[31] Dobson R, Burgess MI, Sprung VS, Irwin A, Hamer M, Jones J, et al. Metabolically healthy and unhealthy obesity: differential effects on myocardial function according to metabolic syndrome, rather than obesity. Int J Obes 2016;40(1):153-61. http: //dx.doi.org/10.1038/ijo.2015.151.

[32] Stefan N, Häring H-U, Hu FB, Schulze MB. Metabolically healthy obesity: epidemiology, mechanisms, and clinical implications.
Lancet Diabetes Endocrinol 2013;1:152-62. http: //dx.doi.org/10.1016/S2213-8587(13)70062-7.

[33] Vachharajani VT, Liu T, Wang X, Hoth JJ, Yoza BK, McCall CE. Sirtuins link inflammation and metabolism. J Immunol Res 2016; 2016:8167273. http://dx.doi.org/10.1155/2016/8167273.

[34] Sun XL, Bu PL, Liu JN, Wang X, Wu XN, Zhao LX. Expression of SIRT1 in right auricle tissues and the relationship with oxidative stress in patients with atrial fibrillation. Xi Bao Yu Fen Zi Mian Yi Xue Za Zhi 2012;28(9):972-4.

[35] Iacobellis G, Zaki MC, Garcia D, Willens HJ. Epicardial fat in atrial fibrillation and heart failure. Horm Metab Res 2014;46(8):587-90. http://dx.doi.org/10.1055/s-0034-1367078.

[36] Fiore D, Gianfrilli D, Giannetta E, Galea N, Panio G, di Dato C, et al. PDE5 inhibition ameliorates visceral adiposity targeting the miR22/SIRT1 pathway: evidence from the CECSID trial. J Clin Endocrinol Metab 2016;101(4):1525-34. http://dx.doi.org/10.1210/ jc.2015-4252.

[37] Song YS, Lee SK, Jang YJ, Park HS, Kim JH, Lee YJ, et al. Association between low SIRT1 expression in visceral and subcutaneous adipose tissues and metabolic abnormalities in women with obesity and type 2 diabetes. Diabetes Res Clin Pract 2013;101(3):341-8. http://dx.doi.org/10.1016/j.diabres.2013.07.002. 